\title{
25. Ergebnisse des Deutschen Freiwilligensurveys 2014: Implikationen für die Engagementpolitik
}

\author{
Clemens Tesch-Römer, Julia Simonson, Claudia Vogel \\ \& Jochen P. Ziegelmann
}

\subsection{Einleitung}

Die Ergebnisse des Deutschen Freiwilligensurveys 2014 zeigen, dass sich heute ein großer Anteil der Wohnbevölkerung Deutschlands im Alter ab 14 Jahren freiwillig engagiert. Der Anteil Engagierter ist in den letzten fünfzehn Jahren deutlich angestiegen, von 34,0 auf 43,6 Prozent. In den letzten fünfzehn Jahren ist die Engagementquote also um insgesamt knapp zehn Prozentpunkte angestiegen. Insbesondere zwischen 2009 und 2014 hat sich dieser Anstieg dynamisiert: Die Quote ist in einem stärkeren Maße als zuvor angestiegen. Die Zunahme des Engagements kann man auf gesellschaftliche Veränderungen, wie beispielsweise die Bildungsexpansion oder die gestiegene Thematisierung des freiwilligen Engagements in Politik und Öffentlichkeit, zurückführen. ${ }^{1}$ Gleichzeitig hat sich das freiwillige Engagement verändert: Es ist vielfältiger geworden, das Tätigkeitsspektrum der Engagierten hat zugenommen, die Organisationsformen haben sich gewandelt und der Zeitaufwand für die Haupttätigkeit ist kleiner geworden. Für die Politik ist über die Frage des Gesamtanteils freiwillig Engagierter hinaus bedeutsam, dass sich Menschen in sehr unterschiedlichen gesellschaftlichen Bereichen engagieren und dass sich

$1 \mathrm{Zu}$ Einflüssen der methodischen Vorgehensweise auf die Höhe der für die jeweiligen Erhebungsjahre ausgewiesenen Engagementquoten siehe Kapitel 2. eine Reihe von Faktoren identifizieren lässt, die freiwilliges Engagement anstoßen.

Verschiedene gesellschaftliche Trends spielen für den Anstieg der Engagementquote eine bedeutsame Rolle, etwa die zunehmende Anzahl von Menschen mit hohem Bildungsabschluss, die gestiegene Erwerbsbeteiligung von Frauen und die gewachsene Zahl von Vereinen und Organisationen, in denen sich Menschen engagieren können. Aber auch die Akteure im Feld der Engagementpolitik haben, über die Ausweitung des gesellschaftlichen Diskurses und die Umsetzung engagementpolitischer Maßnahmen, dazu beigetragen, dass freiwilliges Engagement für viele Menschen wichtiger geworden ist. Die Bundesregierung hat im Jahr 2010 eine Nationale Engagementstrategie beschlossen (Bundesregierung 2010). Aufgrund eines Beschlusses des Deutschen Bundestages vom 19. März 2009 ist die Bundesregierung verpflichtet, einmal pro Legislaturperiode einen wissenschaftlichen Bericht zur Entwicklung des bürgerschaftlichen Engagements in Deutschland vorzulegen. Das Bundesministerium für Familie, Senioren, Frauen und Jugend ist für diese Engagementberichterstattung verantwortlich. Der Erste Engagementbericht erschien im Jahr 2012 (Deutscher Bundestag 2012), der zweite Engagementbericht soll im Jahr 2016 veröffentlicht werden. Alle Bundesländer haben Grundsätze und Maßnahmen zur Förderung des freiwilligen Engagements vorgelegt. Zivil- 
gesellschaftliche Organisationen sind ebenfalls am Diskurs und der Förderung des freiwilligen Engagements beteiligt (stellvertretend Krimmer \& Priemer 2013).

Trotz der überwiegend positiven Befunde geben die Ergebnisse des Freiwilligensurveys 2014 aber auch Hinweise darauf, dass keineswegs alle Menschen am freiwilligen Engagement in gleicher Weise teilhaben können. Vielmehr gibt es einige gesellschaftliche Gruppen, in denen die Beteiligung am freiwilligen Engagement zum Teil erheblich unter dem bundesdeutschen Durchschnitt liegt. Im vorliegenden Kapitel des Berichts zum Freiwilligensurvey 2014 möchten wir die Frage diskutieren, wie gesellschaftlichen Gruppen, die derzeit im freiwilligen Engagement unterrepräsentiert sind, der Zugang zu dieser Form der gesellschaftlichen Teilhabe besser ermöglicht werden könnte als dies bisher der Fall ist.

\subsection{Freiwilliges Engagement als Teilhabe an der Gesellschaft}

Freiwilliges Engagement wird in der öffentlichen und wissenschaftlichen Debatte häufig mit Blick auf seine gesellschaftlichen Funktionen diskutiert: Freiwilliges Engagement trägt zum Zusammenhalt der Gesellschaft bei, treibt die Weiterentwicklung der Demokratie voran und ermöglicht innovative Lösungen für gesellschaftliche Probleme (Olk \& Hartnuß 2011: 5; Zimmer \& Rauschenbach 2011: 11). Freiwilliges Engagement fördert gegenseitiges Vertrauen und dient dazu, unterschiedliche Gruppen in die Gesellschaft zu integrieren. Darüber hinaus gibt es auch Versuche, den ökonomischen Wert des freiwilligen Engagements zu erfassen: Definiert man freiwillige Tätigkeiten als eine Form der Arbeit, so wird es möglich, sie zu klassifizieren und ihren ökonomischen Wert festzustellen (International Labour Organization (ILO) 2011: 5).

Freiwilliges Engagement dient nicht allein dem Wohl der Gesellschaft, sondern tut auch den engagierten Menschen selbst gut. Menschen, die sich freiwillig engagieren, sind über gemeinschaftliche Tätigkeiten eingebunden in die Gesellschaft: Sie lernen andere Freiwillige kennen, erweitern ihr soziales Netzwerk und haben Freude an gemeinsamen Aktivitäten, sie tauschen sich mit anderen Freiwilligen aus und bilden sich im Rahmen ihres Engagements weiter. Aus individueller Sicht ist freiwilliges Engagement also eine Möglichkeit der Vergesellschaftung, der Teilhabe an gesellschaftlichen Willensbildungs- und Entscheidungsprozessen, des Findens von Lebensfreude und Lebenssinn, aber auch, ganz handfest, dem Erwerb von Kompetenzen und der Herstellung von sozialen Beziehungen, die in unterschiedlicher Weise relevant und hilfreich sein können. Während des freiwilligen Engagements können sich berufliche Perspektiven ergeben, sei es über den Erwerb relevanter Kompetenzen oder über den Kontakt zu potenziellen Arbeitgebern. Sich freiwillig zu engagieren kann positiv auf Lebenszufriedenheit und Gesundheit wirken (Morrow-Howell, Hong \& Tang 2009). Die Engagement-Strategie des Bundesministeriums für Familie, Senioren, Frauen und Jugend bezeichnet dieses Ziel sehr klar: „Zivilgesellschaftliches Engagement ist nicht nur freiwillig, es ist ein Recht auf gesellschaftliche Teilhabe, das für alle in Deutschland lebenden Menschen gilt" (BMFSFJ 2016: 4).

Allerdings ist freiwilliges Engagement an Voraussetzungen und Ressourcen geknüpft, die sozial strukturiert und nicht jedem Menschen in gleichem Maße zugänglich sind (Simonson, Hagen, Vogel \& Motel-Klingebiel 2013). Häufig wird freiwilliges Engagement in weiterführenden Schulen eingeübt. Menschen, die einen niedrigen Bildungsabschluss haben und keine weiterführende Schule besucht haben, fehlt möglicherweise diese Einübung in das freiwillige Engagement (Klatt, Walter \& Bebnowski 2011). Für die Ausübung des freiwilligen Engagements 
wird in der Regel körperliche Mobilität vorausgesetzt. Für gesundheitlich eingeschränkte Menschen ist daher eine Beteiligung am freiwilligen Engagement sehr viel schwieriger als für Menschen ohne gesundheitliche Einschränkungen. Für Menschen mit Migrationshintergrund kann eine Beteiligung am freiwilligen Engagement ebenfalls nur eingeschränkt möglich sein, etwa aufgrund sprachlicher Probleme oder kultureller Vorbehalte auf Seiten von Vereinen und Verbänden. Soziale Ungleichheiten können aber nicht nur eine ungleiche Beteiligung im Engagement bewirken, sondern soziale Ungleichheiten können durch Beteiligung am freiwilligen Engagement womöglich noch verstärkt werden (Böhnke 2011). Daher ist es von hoher Bedeutung, jene Gruppen zu betrachten, die sich - verglichen mit dem bundesdeutschen Durchschnitt (Engagementquote: 43,6 Prozent) - zu deutlich geringeren Anteilen freiwillig engagieren. Insgesamt haben wir fünf Gruppen identifiziert, in denen die Anteile freiwillig engagierter Menschen zum Teil deutlich unterdurchschnittlich sind:

- Menschen mit starken gesundheitlichen Einschränkungen - Engagementquote: 25,8 Prozent

- Menschen mit geringer Bildung - Engagementquote: 28,3 Prozent

- Menschen mit Migrationshintergrund Engagementquote: 31,5 Prozent

- Menschen im höheren Lebensalter (65 Jahre und älter) - Engagementquote: 34,0 Prozent

- Frauen - Engagementquote: 41,5 Prozent

Möglicherweise bestehen für jene Bevölkerungsgruppen, die sich aktuell unterdurchschnittlich engagieren, Zugangsbarrieren zum freiwilligen Engagement. Die normative Wendung innerhalb der zweiten Sichtweise auf freiwilliges Engagement (,Freiwilliges Engagement tut den Engagierten gut, aber nicht alle Bevölkerungsgruppen haben den gleichen Zugang zum freiwilligen Engagement') besteht darin, zu fragen, welche Rahmenbedingungen verändert werden könnten, um jenen Bevölkerungsgruppen einen besseren Zugang zum freiwilligen Engagement zu ermöglichen, die diesen aktuell noch nicht haben. Eine Reihe von Akteuren im Feld der Engagementpolitik hat Einfluss auf die Rahmenbedingungen des Engagements:

- Bund: Die Bundesregierung hat es sich zur Aufgabe gemacht, durch geeignete Rahmenbedingungen bürgerschaftliches Engagement $\mathrm{zu}$ unterstützen, nachhaltig zu fördern und eine Kultur der Anerkennung zu schaffen. Die nationale Engagementstrategie der Bundesregierung (Bundesregierung 2010) verfolgt dabei vier Ziele: (a) eine bessere Abstimmung engagementpolitischer Vorhaben von Bundesregierung, Ländern und Kommunen, (b) die Einbindung von Stiftungen und des bürgerschaftlichen Engagements von Wirtschaftsunternehmen, (c) eine größere Anerkennung und Wertschätzung der Leistungen von freiwillig Engagierten sowie (d) bessere Rahmenbedingungen für das freiwillige Engagement. Insbesondere die Rahmenbedingungen für freiwilliges Engagement sind hier von Bedeutung. In der Engagementstrategie des BMFSFJ werden sechs Handlungsfelder genannt: Förderung von Engagementinfrastruktur, Stärkung der Anerkennungskultur, Verstetigung und Weiterentwicklung der Freiwilligendienste, Strukturierung der Engagementforschung, Sicherstellung wirkungsorientierter Engagementförderung sowie Stärkung der öffentlichen Wahrnehmung (BMFSFJ 2016).

- Länder: Auch die Bundesländer gestalten Engagementpolitik und Engagementförderung. Die entsprechenden Maßnahmen der Länder reichen von der Förderung von Infrastruktureinrichtungen (wie etwa Freiwilligenagenturen, Seniorenbüros oder Selbsthilfekontaktstellen), über die Stärkung der Anerkennung 
für Engagement bis zur Förderung von Qualifizierungsmaßnahmen für Engagierte.

- Kommunen: Freiwilliges Engagement findet vor Ort statt. Die Mitwirkung in der lokalen Politik zählt ebenso dazu wie Engagement in lokalen Initiativen und Vereinen (Zimmer \& Backhaus-Maul 2012). Kommunale Akteure spielen eine bedeutsame Rolle bei der Ermöglichung freiwilligen Engagements.

- Organisationen der Zivilgesellschaft: Initiativen, Vereine, Verbände, Stiftungen, soziale Bewegungen, Nichtregierungsorganisationen (NGOs) und Non-Profit-Organisationen (NPOs) bilden die Organisationen der Zivilgesellschaft. In Deutschland agieren zurzeit etwa 600.000 Organisationen der Zivilgesellschaft (Krimmer \& Priemer 2013). Da Organisationen im direkten Kontakt mit freiwillig engagierten Menschen stehen, haben sie einen großen Einfluss auf Art, Ausgestaltung und Stabilität des freiwilligen Engagements.

Bund, Länder, Kommunen und Organisationen der Zivilgesellschaft gestalten, auf unterschiedlichen Ebenen, die Rahmenbedingungen des Engagements in Deutschlands, aber es gibt noch weitere Akteure in diesem Feld, die von Bedeutung sind. Schulen gestalten vielfach Rahmenbedingungen für freiwilliges Engagement. Da Erwerbsarbeit nicht selten Ansatzpunkte für freiwilliges Engagement bietet, sind auch Unternehmen wichtige Akteure, die Engagement durch geeignete Maßnahmen anregen können (z. B. über flexible Arbeitszeitregelungen). Auch die Zusammenarbeit zwischen Haupt- und Ehrenamtlichen ist in diesem Zusammenhang als hoch bedeutsames Handlungsfeld zu nennen (Schumacher, 2015). Auch wenn wir im Folgenden nicht auf alle relevanten Akteure eingehen können, so gelten unsere Argumente für alle Organisationen, Institutionen und Betriebe, die Zugänge $\mathrm{zu}$ freiwilligem Engagement auch für jene gesellschaftlichen Gruppen schaffen können, die diese bislang nicht in ausreichendem Umfang haben.

Wir möchten im Folgenden anhand von Beispielen diskutieren, inwiefern Grundsätze, Politikentwürfe und Maßnahmen von Bund, Ländern, Kommunen und Organisationen der Zivilgesellschaft die oben identifizierten Gruppen berücksichtigen. Damit greifen wir einen einzelnen Aspekt der zahlreichen Befunde heraus, die wir in diesem Bericht vorgelegt haben. Zudem wird es hier nicht möglich sein, eine gründliche Politikfeldanalyse mit Blick auf Maßnahmen zur Gewinnung im freiwilligen Engagement unterrepräsentierter Gruppen vorzunehmen. Wir sind uns auch sicher, dass im lebendigen Diskurs der zivilgesellschaftlichen Akteure vielfältige Impulse für die Förderung freiwilligen Engagements entwickelt werden, die über unsere Überlegungen weit hinausgehen werden. In der Diskussion werden wir die oben identifizierten Gruppen in einer geänderten Reihenfolge betrachten (Frauen, Menschen im höheren Lebensalter, Menschen mit Migrationshintergrund, Menschen mit starken gesundheitlichen Einschränkungen sowie Menschen mit geringer Bildung).

\subsection{Zugang zum freiwilligen Engagement für Frauen}

Frauen engagieren sich mit 41,5 Prozent seltener als Männer mit 45,7 Prozent. Frauen haben ihr Engagement zwischen 1999 und 2014 deutlicher ausgeweitet als Männer und sich damit den Quoten der Männer angenähert. Die Unterschiede im freiwilligen Engagement von Frauen und
Männern zeigen sich insbesondere in der Ausgestaltung des freiwilligen Engagements (siehe Kapitel 24). Freiwillig engagierte Frauen üben zum Beispiel nach wie vor seltener eine Leitungsoder Vorstandsfunktion im Engagement aus. Gleichstellungspolitische Maßnahmen können 
dazu beitragen, dem Ziel der Gleichstellung von Frauen und Männern im freiwilligen Engagement etwas näher zu kommen.

- Bund: Die Gleichstellungspolitik des Bundes schließt auch den Aspekt der Gleichstellung von Frauen und Männern im Engagement ein. Die systematische Überprüfung von Geschlechterdifferenzen und Herstellung von Geschlechtergerechtigkeit (Gender Mainstreaming) ist hierfür ein etabliertes Vorgehen (BMFSFJ 2015).

- Länder: Auch die Länder machen Gleichstellungspolitik, in Brandenburg zum Beispiel ist die Landesgleichstellungsbeauftragte beim Ministerium für Arbeit, Soziales, Gesundheit, Frauen und Familie angesiedelt. Ihre Arbeit hat unter anderem zum Ziel, Maßnahmen für den „Abbau von Benachteiligungen aufgrund des Geschlechts im sozialen, wirtschaftlichen, rechtlichen und administrativen Bereich" sowie „zur Förderung der gesellschaftlichen Partizipation" durchzuführen (Ministerium für Arbeit, Soziales, Gesundheit, Frauen und Familie, Brandenburg 2015).

- Kommunen: In den Gemeinden spielen Informations- und Kontaktstellen für Engagierte und Interessierte eine zunehmend größere Rolle. Auch bei der Vermittlung von freiwilligen Tätigkeiten sollten Mitarbeiterinnen und Mitarbeiter von Informations- und Kontaktstellen für die Themen Geschlechterstereotype und Geschlechterungleichheit sensibilisiert werden (Stichwort: Gender Mainstreaming).

- Organisationen der Zivilgesellschaft: Angesichts der niedrigeren Engagementbeteiligung der Frauen sind Organisationen gut beraten, Frauen wie Männer gleichermaßen auf eine Engagementbeteiligung anzusprechen, um die im Ersten Gleichstellungsbericht der Bundesregierung kritisierte Reproduktion der persistenten Rollenmuster im Engagement aufzubrechen (BMFSFJ 2013: 186). Um die Gleichstellung im freiwilligen Engagement voranzubringen, sollten Organisationen gezielt um Frauen als Engagierte werben. Insbesondere sollten die Organisationen Frauen ebenso häufig wie Männern Leitungsfunktionen und Führungsaufgaben im freiwilligen Engagement übertragen. In vielen Organisationen ist Gleichstellung als wichtiges Thema längst erkannt und institutionell verankert. Beispiele aus dem Deutschen Olympischen Sportbund (DOSB), der auch eine Vizepräsidentin für Frauen und Gleichstellung hat, sind die DOSB-Frauen-Vollversammlung sowie die 2014 beschlossene Geschlechterquote: In allen Gremien des DOSB müssen künftig Frauen und Männer zu jeweils mindestens 30 Prozent vertreten sein (Deutscher Olympischer Sportbund (DOSB) 2015a). Ein weiterer Ansatz ist die Gewinnung und Ausbildung von Frauen als Trainerinnen im Projekt „Stärkung des Trainerberufes mit dem Ziel der Gleichstellung“. So sollen mehr angestellte, aber insbesondere auch mehr ehrenamtliche Trainerinnen beteiligt werden.

Der Erste Gleichstellungsbericht nennt Bereiche, in denen „die Rückstände Deutschlands zu anderen Ländern eher gestiegen sind“ (BMFSFJ 2013: 45), wie beispielsweise „die Fragmentierung weiblicher Erwerbstätigkeit“ (ebd.). Insofern sollte man also nicht nur auf positive Transfereffekte hoffen (Gleichstellung in der Erwerbstätigkeit begünstigt Gleichstellung im Engagement), sondern man sollte direkt im Engagementfeld an einer Verbesserung der Gleichstellung arbeiten. Diese muss eingebettet sein in einen Diskurs um eine Neuverhandlung der gesamtgesellschaftlichen Arbeitsteilung zwischen Frauen und Männern in allen für die Wohlfahrtsproduktion relevanten gesellschaftlichen Sektoren, das heißt in Familie, Markt, Staat und Zivilgesellschaft. 


\subsection{Zugang zum freiwilligen Engagement für Menschen im höheren Lebensalter}

Betrachtet man die Anteile freiwillig Engagierter über die Lebensphasen, so erkennt man, dass der Anteil engagierter Menschen im höheren Lebensalter abnimmt. Allerdings ist dieser Rückgang vor allem auf die Menschen von 70 Jahren und älter zurückzuführen - auf jene Menschen also, die sich an der Schwelle zum hohen Alter befinden. Bis zu einem Alter von 70 Jahren ist der Anteil engagierter Menschen recht stabil (50-54 Jahre: 46,1 Prozent, 55-59 Jahre: 44,5 Prozent, 60-64 Jahre: 45,9 Prozent, 65-69 Jahre: 43,7 Prozent, 70-74 Jahre: 39,9 Prozent, 75 Jahre und älter: 26,1 Prozent). Anzumerken ist allerdings auch, dass das Engagement älterer Menschen im Vergleich zum Engagement der mittleren Altersgruppen in den letzten fünfzehn Jahren stärker zugenommen hat. Zudem zeigen Analysen zur biografischen Dauer des Engagements, dass Menschen auch im höheren Lebensalter noch mit einer neuen freiwilligen Tätigkeit beginnen. Es lässt sich also feststellen, dass freiwilliges Engagement im ,dritten Lebensalter' mittlerweile recht weit verbreitet ist, dass aber im ,vierten Lebensalter' die Beteiligung am freiwilligen Engagement deutlich nachlässt.

- Bund: Seit mehreren Jahren gibt es auf der Bundesebene eine klare Positionierung für die Unterstützung eines aktiven Alters. $\mathrm{Zu}$ erkennen ist dies beispielsweise am Fünften und Sechsten Altenbericht. Im Fünften Altenbericht stellt die Bundesregierung fest, dass es notwendig ist, „förderliche Rahmenbedingungen für freiwilliges Engagement im Alter und insbesondere eine das Engagement unterstützende Infrastruktur zu schaffen “ (BMFSFJ 2005). Eine Reihe von Maßnahmen können hier genannt werden, die von der Bundesregierung in den vergangenen Jahren gefördert wurden, etwa die seit zwei Jahrzehnten unterstützte Bundesarbeitsgemeinschaft Seniorenbüros, das Bundesmodellprogramm
,Erfahrungswissen für Initiativen 'sowie das ,Aktionsprogramm Mehrgenerationenhäuser. Mit Blick auf den im höheren Lebensalter festzustellenden Rückgang in der Teilnahme am freiwilligen Engagement kommt der Sechste Altenbericht $\mathrm{zu}$ dem Schluss, „dass Teilhabe- und Engagementdefizite in vielen Fällen nicht auf ein individuelles Rückzugsbedürfnis, sondern vielmehr auf unzureichende Teilhabemöglichkeiten und Altersbilder, die Verluste akzentuieren, zurückgehen" (BMFSFJ 2013: 22). Zudem betont der Sechste Altenbericht im Jahr 2010 die Notwendigkeit einer Anpassung der Altersbilder des vierten Lebensalters, in dem die Vielfalt menschlichen Alterns genauso zum Ausdruck kommt, wie die Aspekte der Inklusion und Teilhabe. Zentral ist hierbei auch eine neue Qualität des Aktivitätsbegriffs, da derzeitig vorherrschende Definitionen des aktiven Alterns der Lebenswelt der Hochaltrigen nicht im vollen Umfang gerecht werden.

- Länder: Auch auf Landesebene sind ältere Menschen eine wesentliche Zielgruppe der Engagementförderung. So heißt es etwa in dem Grundsatzpapier des ,Runden Tisches Bürgerschaftliches Engagement ${ }^{\star}$ der Bayerischen Staatsregierung: „Das Erfahrungswissen älterer Menschen gilt es den nachfolgenden Generationen zu erschließen (z. B. Mentoren-, Patenschaftsprojekte)“ (Bayerisches Staatsministerium für Arbeit und Soziales, Familie und Integration 2010: 15). Dabei wird auch die Umsetzung der Bundesmodellprogramme ,Mehrgenerationenhäuser' und ,Erfahrungswissen für Initiativen' in Bayern hervorgehoben. Allerdings wird auch darauf verwiesen, dass ältere Menschen nicht allein potenziell Engagierte sind, sondern, insbesondere im hohen Alter, Zielgruppen des Engagements. So heißt es in dem angesprochenen Grundsatzpapier: „Pfle- 
ge, Demenz und Altersarmut etwa werden in den kommenden Jahren große Themenfelder Bürgerschaftlichen Engagements sein. Es gilt, diesbezüglich frühzeitig neue Wege des Engagements zu erproben, zu entwickeln und zu etablieren" (Bayerisches Staatsministerium für Arbeit und Soziales, Familie und Integration 2010: 15).

- Kommunen: Die von Bund und Ländern geförderten Programme haben Strukturen auf kommunaler Ebene entstehen lassen. Neben der herkömmlichen Vereinsförderung ist, beispielsweise mit Freiwilligenagenturen, Mehrgenerationenhäusern und Seniorenbüros ein vielfältiges Spektrum an engagementfördernden Infrastrukturen entstanden (Jakob 2010). In der fachlichen Diskussion dieser engagementfördernden Infrastrukturen steht jedoch die Frage der Finanzierung, Vernetzung und Einbindung neuer Akteure im Mittelpunkt und weniger die Frage, ob jene Gruppen, die wie ältere Menschen im freiwilligen Engagement unterrepräsentiert sind, durch diese kommunalen Infrastrukturmaßnahmen angesprochen werden.

- Organisationen der Zivilgesellschaft: Mit ihren Tätigkeiten und Angeboten richten sich Organisationen der Zivilgesellschaft an un- terschiedliche Altersgruppen. Die ZiviZ-Erhebung ,Zivilgesellschaft in Zahlen' hat gezeigt, dass die große Mehrzahl der Organisationen (etwa drei Viertel) sich an alle Altersgruppen wenden, etwa zehn Prozent an Menschen über 65 Jahre und 15 Prozent an Kinder und Jugendliche (Krimmer \& Priemer 2013: 29). Offen ist allerdings die Frage, wie es zivilgesellschaftlichen Organisationen gelingen könnte, Angebote für Menschen bis ins hohe Alter zu machen.

Im gesellschaftlichen und wissenschaftlichen Diskurs wird oft angeführt, dass sich ältere Menschen trotz ihres ,Zeitwohlstandes' in geringerem Umfang engagieren als jüngere Menschen. Bedenkt man hierbei, dass es noch immer an Engagementmöglichkeiten mangelt, die auf die Bedürfnisse der Älteren zugeschnitten sind (Dathe 2011), so ist die aktuelle Engagementbeteiligung der Älteren durchaus beachtlich. Diese Beteiligung kann durch eine vermehrte Bereitstellung von auf Bedürfnisse der Älteren zugeschnittenen Engagementmöglichkeiten gesteigert werden. Auch für ältere Menschen mit gesundheitlichen Einschränkungen oder solche, die pflegebedürftig sind, sollten die Möglichkeiten für eine „selbstbestimmte und gestaltende Teilhabe“ offen stehen, wenn sie eine solche Teilhabe wünschen (Alisch \& Kümpers 2015: 5).

\subsection{Zugang zum freiwilligen Engagement für Menschen mit Mitgrationshintergrund}

Die Ergebnisse des Freiwilligensurveys zeigen ganz deutlich, dass Menschen mit Migrationshintergrund keine homogene Gruppe sind, sondern sich beispielsweise je nach Biografie und Migrationsgeschichte ganz unterschiedlich engagieren: Während sich Personen mit Migrationshintergrund, aber ohne eigene Zuwanderungserfahrung zu recht ähnlichen Anteilen freiwillig engagieren wie Personen ohne Migrationshinter- grund, sind die Engagementquoten bei Personen mit Zuwanderungserfahrung noch unterdurchschnittlich. Aufgrund der hohen Engagementbereitschaft besteht hier jedoch auch ein hohes Potenzial. Dies bedeutet aber auch, dass es ganz unterschiedliche, zielgruppenspezifische Unterstützungsmaßnahmen für diese Gruppen geben sollte. 
- Bund: Der Zustrom von Schutzsuchenden aus Syrien und anderen Krisengebieten Ende 2015 stellt nicht nur eine große Herausforderung für Politik und Zivilgesellschaft in Deutschland dar, er könnte auch ohne die Arbeit vieler ehrenamtlicher Helferinnen und Helfer nicht bewältigt werden. Ganz aktuell zeigt dieses Thema, wie bedeutsam es ist, in Deutschland lebende Menschen mit Migrationshintergrund nicht allein als Personenkreis zu betrachten, die auf das Engagement von andern angewiesen sind, sondern auch als Personenkreis, in dem ein großes Potenzial für die Übernahme freiwilliger Arbeiten und Aufgaben besteht. Die Bundesregierung hat darauf reagiert und das Sonderprogramm ,Bundesfreiwilligendienst mit Flüchtlingsbezug' geschaffen, bereits ab Dezember 2015 stehen die ersten der 10.000 zusätzlichen Bundesfreiwilligendienstplätze zur Verfügung. Besonders integrativ scheint an diesem Programm, dass auch Asylberechtigte daran teilnehmen können (BMFSFJ 2015). Für Personen ohne ausreichende deutsche Sprachkenntnisse sind bei Bedarf vierwöchige Intensivsprachkurse vorgesehen. $\mathrm{Ob}$ ausreichend Personen mit eigener Zuwanderungserfahrung für den Dienst gewonnen werden können, bleibt abzuwarten. Eine unterstützende Maßnahme ist das Patenschaftsprogramm "Menschen stärken Menschen" des Bundesministeriums für Familie, Senioren, Frauen und Jugend, das folgende Maßnahmen beinhaltet: Aufbau von Patenschafts-Tandems, Wissenstransfer zwischen Programmträgern sowie Weiterentwicklung von Qualitätsstandards für Patenschaftsprogramme.

- Länder: Welche Bedeutung das freiwillige Engagement für die Integration von Menschen mit Migrationshintergrund haben kann, ist auch von vielen Landesregierungen bereits erkannt worden. Baden-Württemberg zum Beispiel hat ein Ministerium für Integration, das neben Einbürgerungskampagnen und Arbeitsmarktpartizipation auch die zivilgesellschaftliche Teilhabe durch eine Vielzahl von Projekten fördert. Dies kommt auch in einer aktuellen Gesetzesinitiative der Landesregierung, $\mathrm{Ge}$ setz zur Verbesserung von Chancengerechtigkeit und Teilhabe in Baden-Württemberg zum Ausdruck (Landtag Baden-Württemberg 2015). In dem Gesetzesvorhaben ist unter anderem vorgesehen, die interkulturelle Öffnung der Landesverwaltung voranzubringen und eine paritätische Besetzung von Menschen mit und ohne Migrationshintergrund in den politischen Entscheidungsgremien zu fördern. Ein solches Gesetz zur Stärkung der Chancengerechtigkeit und Teilhabe kann dazu beitragen, auch die Zugangschancen von Migrantinnen und Migranten im freiwilligen Engagement zu verbessern. Auch in anderen Bundesländern, zum Beispiel in Sachsen, wird auf die interkulturelle Öffnung etwa der Verwaltung gesetzt, aber laut Koalitionsvertrag auch darauf, die Bildung von ,Parallelgesellschaften' zu verhindern (CDU Sachsen \& SPD Sachsen 2014).

- Kommunen: In Deutschland existiert heute eine Vielzahl von Migrantenorganisationen, die wichtige Akteure für die Förderung des Engagements für Menschen mit Migrationshintergrund sind. Auf der kommunalen Ebene hängen Erfolg und Misserfolg von Migrantenorganisationen davon ab, ob sie Zugang zu den regionalen Netzwerken haben und ob sie Zugang zu Fördermitteln haben (Weiss 2013). Am Beispiel der Stadt Bremen konnten Habermann und Stagge (2014) zeigen, dass die Entwicklung niedrigschwelliger Angebote für ältere Menschen mit Migrationshintergrund sozialraumbezogener Steuerung durch die kommunalen Akteure und Kooperation mit den beteiligten Organisationen und den Bürgerinnen und Bürgern in den Stadtteilen erfordert, da ältere Migrantinnen und Migranten bei den Regelangeboten der kommunalen $\mathrm{Al}$ tenhilfearbeit bislang unterrepräsentiert sind. Das bedeutet auch, dass gemeinsame Netzwerke etwa mit etablierten Wohlfahrtsverbänden und den Migrantenorganisationen geschaffen werden müssen. Bezüglich kommunaler 
Aufgaben wie der Arbeit der Freiwilligen Feuerwehr wird etwa vom Vizepräsidenten des Deutschen Feuerwehrverbandes darüber geklagt, es gäbe Probleme bei der Gewinnung von Ehrenamtlichen (Deutscher Bundestag 2015). Abhilfe schaffen könnte hier die interkulturelle Öffnung der Freiwilligen Feuerwehr und die Ermöglichung des Einstiegs in die Feuerwehrarbeit als Erwachsener (neben den klassischen Wegen über die Kinder- und Jugendfeuerwehren), so könnten auch mehr Menschen mit Zuwanderungsgeschichte gewonnen werden.

- Organisationen der Zivilgesellschaft: Eine wichtige Aufgabe der zivilgesellschaftlichen Organisationen ist die interkulturelle Öffnung, um Menschen mit und Menschen ohne Migrationshintergrund gleichermaßen für ihre Angebote zu erreichen. Im Bereich Sport finden sich hierfür zahlreiche vorbildhafte Beispiele, denn der Sport ist nicht nur im Leistungsund Spitzensport international ausgerichtet. Das Programm ,Integration durch Sport' des Deutschen Olympischen Sportbundes besteht bereits seit 25 Jahren (Deutscher Olympischer Sportbund (DOSB) 2015b). Es hat die Zielstellung, Menschen mit Migrationshintergrund für den Vereinssport zu werben, Ehrenamtliche aus den Reihen der Menschen mit Migrationshintergrund $\mathrm{zu}$ gewinnen sowie Sport und Vereine interkulturell zu öffnen. Wichtig ist hierbei, dass interkulturelle Öffnung nicht nur auf die Ansprache und Gewinnung von Menschen mit Migrationshintergrund für die Organisationen zielt (etwa durch fremdsprachige Flyer oder die Ausbildung von Übungsleiterinnen und Übungsleitern, die selbst einen Migrationshintergrund haben), sondern auch auf die Qualifizierung aller Vereinsmitglieder im Bereich der interkulturellen Öffnung.

Am Beispiel der Migrantinnen und Migranten wird deutlich, dass sich eine differenzierte Betrachtung spezifischer Bevölkerungsgruppen lohnt. Daher sollten auch Maßnahmen, das Engagement $\mathrm{zu}$ fördern, genau auf die spezifischen Gruppen zugeschnitten werden. Mit Blick auf Anerkennungs- und Wertschätzungskultur freiwilligen Engagements ist $\mathrm{zu}$ fragen, ob es hilfreich ist, das Engagement in Migrantenselbstorganisationen als Gefahr für die Integration und im „Zusammenhang mit Abschottungstendenzen und Parallelgesellschaften“ zu diskutieren (Rindt, Klein \& Klein 2011: 6). Möglicherweise ist es sinnvoller, auch diesen Formen von Engagement öffentliche Wertschätzung entgegenzubringen. Bisher nicht engagierten, aber zum Engagement bereiten Menschen (z. B. Personen mit Zuwanderungserfahrung) könnte auf diese Weise das Mitmachen in Organisationen leichter gemacht werden.

\subsection{Zugang zum freiwilligen Engagement für Menschen mit gesundheitlichen Einschränkungen}

Menschen mit krankheitsbedingten Einschränkungen engagieren sich zu geringeren Anteilen als Menschen ohne Erkrankungen beziehungsweise ohne krankheitsbedingte Alltagseinschränkungen. Die Engagementquote liegt bei Menschen, die durch Krankheit in der Ausübung ihrer alltäglichen Arbeiten stark eingeschränkt sind, bei 25,8 Prozent und bei Menschen, die krankheitsbedingt etwas eingeschränkt sind, bei 38,7 Prozent. Demgegenüber liegen die Engagementquoten bei Menschen, die krankheitsbedingt nicht eingeschränkt sind bei 47,4 Prozent, sowie bei Personen, die keine Erkrankung haben, bei 48,0 Prozent. Ähnliche Unterschiede findet man zwischen Gruppen unterschiedlich guter subjektiver Gesundheit: Menschen mit schlechter 
subjektiver Gesundheit engagieren sich zu geringeren Anteilen als Menschen mit guter subjektiver Gesundheit. In höheren Altersgruppen ist der Anteil an Engagierten bei Personen mit starken krankheitsbedingten Alltagseinschränkungen besonders niedrig (18,3 Prozent). Bei Menschen mit hoher Bildung wirken sich Alltagseinschränkungen weniger stark auf die Wahrscheinlichkeit aus, engagiert zu sein (38,9 Prozent).

- Bund: Die Integration und Inklusion von Menschen mit Behinderungen und krankheitsbedingten Alltagseinschränkungen sind ein wichtiges politisches Ziel auf Bundesebene. Allerdings geht es dabei häufig eher um die Frage, wie freiwillig Engagierte Menschen mit Behinderung in ihrer Lebensführung unterstützen können. Mit Blick auf das ,Freiwillige Soziale Jahr' wird betont, dass Freiwillige mit ihrem Engagement $\mathrm{zu}$ einer besseren gesellschaftlichen Teilhabe und Integration von Menschen mit Behinderung beitragen. Allerdings ist in den letzten Jahren auch auf Bundesebene das freiwillige Engagement von Menschen mit Behinderungen in den Fokus gerückt. Mit dem im Jahr 2013 erschienenen ,Teilhabebericht der Bundesregierung über die Lebenslagen von Menschen mit Beeinträchtigungen' hat die Bundesregierung begonnen, auch das freiwillige Engagement von Menschen mit Behinderungen in den Blick zu nehmen (Bundesministerium für Arbeit und Soziales (BMAS) 2013: 247ff.).

- Länder: Auf Landesebene gibt es eine Reihe von Initiativen und Maßnahmen, in denen es um den Einbezug von Menschen mit Behinderungen und krankheitsbedingten Beeinträchtigungen geht. Beispielsweise zielt das Hamburger Projekt „Selbstverständlich Freiwillig“ darauf $a b$, dass sich Menschen mit Behinderungen besser als bisher engagieren können (Habenicht \& Pieper 2013). In diesem Projekt engagieren sich nicht Freiwillige für Menschen mit Behinderungen, sondern Menschen mit Behinderungen, die etwas für sich und andere tun wollen. Ein wichtiger Punkt ist dabei das Umdenken auf beiden Seiten: Menschen mit Behinderungen lernen, sich selbst als Handelnde zu sehen - und Freiwilligenorganisationen bemühen sich darum, die Mitarbeit von Menschen mit Behinderungen möglich zu machen, und zwar da, wo ihre „Interessen liegen, und da, wo es gesellschaftlich sichtbar wird: Zum Beispiel in der Kirchengemeinde, beim Motorradgottesdienst, in der Seniorenarbeit, bei kulturellen Aktivitäten, in der Obdachlosenhilfe oder im Naturschutz" (Habenicht \& Pieper 2013: 8).

- Kommunen: Kommunen sind Lebensorte für Menschen mit (und ohne) Behinderungen oder Alltagseinschränkungen. Daher ist die Frage, wie Menschen mit Behinderungen der Zugang zum freiwilligen Engagement ermöglicht werden kann, auch für Kommunen von großer Bedeutung. Ein möglicher Ansatzpunkt sind hierbei verstärkte Informationen über die besonderen Bedarfe von Menschen mit Behinderungen oder Alltagseinschränkungen sowie die Vernetzung verschiedener kommunaler Akteure. Bislang sind aber nur wenige kommunalpolitische Entwürfe für die verstärkte Inklusion von Menschen mit Behinderung in das freiwillige Engagement zu finden.

- Organisationen der Zivilgesellschaft: Spricht man über die Beteiligung von Menschen mit Behinderungen und krankheitsbedingten Alltagseinschränkungen in Organisationen der Zivilgesellschaft, so richtet sich der Blick häufig auf Organisationen der Selbsthilfe. Die dabei zugrundeliegende Überlegung lautet, dass sich Betroffene am ehesten mit anderen Betroffenen zusammenschließen. Daher gibt es eine Reihe von Informationen zur Beteiligung von Menschen mit Behinderungen und krankheitsbedingten Alltagseinschränkungen im Bereich der Selbsthilfe (Matzat 2010). Die Beteiligung von Menschen mit Behinderungen und Alltagseinschränkungen in der re- 
gulären Freiwilligenarbeit wird dagegen eher selten thematisiert.

Menschen mit Behinderungen oder krankheitsbedingten Alltagseinschränkungen benötigen für das tägliche Leben vielfach selbst Unterstützung. Diese Personengruppe als freiwillig Engagierte wahrzunehmen und zu unterstützen, macht einen Perspektivwechsel notwendig. Grundlage dafür ist unter anderem die UN-Konvention über die Rechte von Menschen mit Behinderung, die Ende 2006 von der Generalversammlung der Vereinten Nationen (United Nations, UN) verabschiedet wurde. Diese Konvention fordert Inklusion, also die gleichberechtigte Teilhabe aller Menschen am gesellschaftlichen Leben. Im Bereich des freiwilligen Engagements sind Menschen mit Behinderungen oder Alltagseinschränkungen derzeit aber entweder Zielgruppen freiwilligen Engagements oder ihnen werden mehr oder weniger explizit spezielle Organisationen zugewiesen (,Selbsthilfe'). Eine stärkere Öffnung des freiwilligen Engagements für Menschen, die in verschiedener Hinsicht eingeschränkt oder behindert sind, ist eine Aufgabe, die in Zukunft erheblicher Anstrengungen bedarf.

\subsection{Zugang zum freiwilligen Engagement für Menschen mit niedriger Bildung}

Menschen mit niedriger Schulbildung engagieren sich im Jahr 2014 zu einem deutlich niedrigeren Anteil (28,3 Prozent) als Personen mit mittlerer und hoher Schulbildung (41,1 Prozent beziehungsweise 52,3 Prozent). Zudem haben die Unterschiede zwischen den Bildungsgruppen zwischen 1999 und 2014 deutlich zugenommen: Während in der Gruppe der Menschen mit hoher Bildung der Anteil der Engagierten von 40,4 Prozent auf 52,3 Prozent stieg (also um 11,9 Prozentpunkte), wuchs der Anteil der Engagierten in der Gruppe der Menschen mit geringer Bildung nur um 3,8 Prozentpunkte, von 24,5 Prozent (im Jahr 1999) auf 28,3 Prozent (im Jahr 2014). Personen mit niedrigerem Bildungsstand gehören, im Vergleich zu Menschen mit mittlerer und höherer Bildung, auch häufiger zu jenen Menschen, die früher einmal engagiert waren, ihr Engagement aber irgendwann im Lebenslauf abgebrochen haben. Auch wenn jene Personen in den Blick genommen werden, die noch nie in ihrem Leben engagiert waren, sticht eine Gruppe besonders hervor: 46,7 Prozent der Personen mit niedriger Bildung waren noch nie freiwillig engagiert - auch dies ist ein weit überdurchschnittlicher Wert. Die Bereitschaft, sich künftig zu engagie- ren, ist bei Niedriggebildeten ebenfalls deutlich weniger ausgeprägt als bei nicht-engagierten Personen mit mittlerer oder hoher Bildung. Neben der Bildung sind weitere sozioökonomische Ressourcen von Bedeutung: Personen, die ihre finanzielle Lage als sehr schlecht einschätzen, engagieren sich zu einem weit geringeren Anteil als Personen, die ihre finanzielle Lage als sehr gut einschätzen.

- Bund: In der Nationalen Engagementstrategie der Bundesregierung (Bundesregierung 2010) wird angemerkt, dass die Engagementförderung in Deutschland heute vor der Herausforderung steht, jenen „Teil der Bevölkerung, der dem Engagement noch fernsteht, besser über die Vielfalt interessanter Engagementmöglichkeiten zu informieren, um das vorhandene Potenzial auch in die Tat umzusetzen" (Abschnitt A, S. 8). Als Ziel wird dabei formuliert: „Wir wollen das Engagement von allen Bevölkerungsgruppen fördern und uns dabei einzelnen Gruppen und Menschen, die am Rande der Gesellschaft stehen, in besonderer Weise widmen. Engagementförderung muss gerade dort ansetzen, wo einzelne Gruppen unterre- 
präsentiert oder ihre Zugänge zum Engagement besonders schwierig sind" (Abschnitt B, S. 11). Auch in der Engagementstrategie des Bundesministeriums für Familie, Senioren, Frauen und Jugend (BMFSFJ) geht es darum, verstärkt nach Mitteln und Wegen zu suchen, die das Engagement von bildungsfernen Schichten fördern (BMFSFJ 2016: 9). Beispiele für Maßnahmen beziehen sich insbesondere auf die Rolle des freiwilligen Engagements für erwerbslose Menschen. Andererseits werden aber Personen mit geringer Bildung weniger als potenzielle Freiwillige, sondern eher als Zielgruppe für das Engagement Anderer gesehen. Möglicherweise ist es sinnvoll, auch Maßnahmen zu entwickeln, in denen Menschen mit geringer Bildung als Freiwillige angesprochen werden und von der Teilhabe am freiwilligen Engagement profitieren. Um soziale Ungleichheit nicht noch zu verschärfen, ist es wichtig, dass Maßnahmen der Engagementförderung auch diejenigen erreichen, bei denen das Engagement am niedrigsten ausgeprägt ist (Schäfer \& Schoen 2013).

- Länder: Auf Länderebene werden häufig Informations- und Kontaktstellen als Angebote der Engagementförderung entwickelt. Obwohl diese Strukturen wichtig für die Aufnahme eines Engagements sind, werden sie in der Regel eher von jenen Personengruppen angenommen, die bereits zu einem hohen Anteil freiwillig engagiert sind. Daher bedarf es besonderer Anstrengungen, insbesondere Menschen mit niedriger Bildung für das Engagement zu gewinnen. Am Beispiel des Landes Baden-Württemberg kann dies veranschaulicht werden. In der ,Engagementstrategie Baden-Württemberg' (Ministerium für Arbeit und Sozialordnung, Familie, Frauen und Senioren 2014) heißt es etwa: „Im Mittelpunkt aller Überlegungen steht die Frage, was die Menschen vor Ort - und zwar bezogen auf ihr jeweiliges regionales, soziales und persönliches Umfeld - brauchen, damit sie sich bürgerschaftlich engagieren können“ (ebd.: 6).
Eindrucksvoll wird eine solche, auf die Bedarfe potenziell engagierter Menschen zielende Strategie für fünf gesellschaftliche Gruppen entwickelt: Menschen mit Migrationshintergrund, pflegende Angehörige, Menschen mit Behinderungen sowie alte und junge Menschen. Die Bedeutung des Bildungsstandes für den $\mathrm{Zu}$ gang zum Engagement wird zwar thematisiert („Fragen der Bildung spielen eine entscheidende Rolle, wenn es um die Voraussetzung für die Aufnahme von Formen Bürgerschaftlichen Engagements geht", ebd.: 47), aber es werden keine systematischen Zugangswege für diese Gruppe entwickelt.

- Kommunen: Engagementpolitik ist eine zentrale Komponente kommunaler Selbstverwaltung. Wird betrachtet, in welcher Form Kommunen Engagementpolitik betreiben, so wird deutlich, dass dies in erster Linie in der finanziellen, sachlichen und personellen Unterstützung von Vereinen und Verbänden geschieht (Zimmer \& Backhaus-Maul 2012). Die Ermöglichung des Zugangs von Menschen mit niedriger Bildung zum freiwilligen Engagement ist dabei eher eine indirekte Folge kommunaler Engagementpolitik.

- Organisationen der Zivilgesellschaft: Um soziale Ungleichheiten abzubauen, könnte es auch eine Aufgabe zivilgesellschaftlicher Organisationen sein, sich verstärkt an Personen mit niedriger Bildung zu richten. Betrachtet man den Bericht über zivilgesellschaftliche Organisationen (Krimmer \& Priemer 2013), so stellt man fest, dass das Stichwort ,Bildung beinahe auf jeder Seite des Berichts zu finden ist. Allerdings gibt es keinen Hinweis darauf, dass zivilgesellschaftliche Organisationen Bildungsangebote insbesondere für Menschen mit niedriger Bildung zu machen. Dies könnte aber bedeuten, dass von Bildungsangeboten und -maßnahmen insbesondere jene Personen profitieren, die bereits über eine gute Bildung verfügen - und sich damit bildungsbedingte Ungleichheiten eher verstärken. 
Akteure der Engagementpolitik - Bund, Länder, Kommunen und zivilgesellschaftliche Organisationen - stellen die benachteiligte Situation von Menschen mit niedriger Bildung deutlich heraus. Im Grundsatz stimmen alle Akteure überein, dass auch und gerade bildungsfernen Menschen der Zugang zum freiwilligen Engagement ermöglicht werden sollte, da Engagement auch Lernerfahrungen ermöglicht. Es fehlen allerdings konkrete und erprobte Maßnahmen, wie dieses Ziel zu erreichen ist. Der Zugang zu guter Bildung für alle ist zwar eine Schlüsselfunktion für die gesellschaftliche Teilhabe (Bundesministerium für Bildung und Forschung (BMBF) 2014), aber es erscheint durchaus sinnvoll, die Beteiligung am freiwilligen Engagement auch als Zugang zu Bildungsmöglichkeiten zu sehen und entsprechend gerade für Menschen mit niedriger Bildung zu fördern.

\subsection{Ausblick}

Ein großer Anteil der Menschen in Deutschland engagiert sich freiwillig, und dieser Anteil Engagierter ist in den letzten fünfzehn Jahren deutlich angestiegen. Begreift man freiwilliges Engagement als Möglichkeit zur gesellschaftlichen Teilhabe, so ist dies durchaus als positiver Befund $\mathrm{zu}$ werten. Allerdings geben die Ergebnisse des Freiwilligensurveys 2014 auch Hinweise darauf, dass keineswegs alle Menschen am freiwilligen Engagement in gleicher Weise teilhaben können. Vielmehr gibt es einige gesellschaftliche Gruppen, in denen die Beteiligung am freiwilligen Engagement zum Teil erheblich unter dem bundesdeutschen Durchschnitt liegt: Frauen, ältere Menschen, Menschen mit gesundheitlichen Einschränkungen, Menschen mit Migrationshintergrund sowie Personen mit geringer Bildung engagieren sich zu unterdurchschnittlichen Anteilen. Darüber hinaus unterscheidet sich die Ausgestaltung des Engagements, insbesondere der Zugang zu prestigeträchtigen Formen des Engagements in Leitungs- und Vorstandsfunktionen bleibt diesen Personengruppen oftmals verwehrt.

Überlagern sich Risikokonstellationen, etwa wenn Frauen eine niedrige Bildung oder gesundheitliche Einschränkungen haben, so können die Zugangschancen zum freiwilligen Engagement besonders deutlich eingeschränkt sein. Diese Wechselwirkung zwischen Ungleichheitsdimensionen wird in der Debatte zu sozialen Ungleich- heiten meist als Intersektionalität beschrieben, die über die reine Kumulation von einzelnen Ungleichheiten hinausgeht (Winker \& Degele 2009).

Um soziale Ungleichheit nicht noch zu verschärfen ist es wichtig, dass Maßnahmen der Engagementförderung auch diejenigen erreichen, bei denen das Engagement nur gering ausgeprägt ist. Angehörige von ,Risikogruppen ' - ältere Menschen, Menschen mit gesundheitlichen Einschränkungen, Menschen mit Migrationshintergrund, Personen mit geringer Bildung - werden in der Engagementförderung häufig als Zielgruppe für das Engagement Anderer gesehen. Freiwillig Engagierte nehmen Angehörige dieser Gruppen als ,Zielobjekte in den Blick. Unter dem Gesichtspunkt, dass Menschen auch die aktive Teilhabe am freiwilligen Engagement nutzen können, erscheint es sinnvoll, bei der Engagementförderung stärker auf die Mitwirkung von Frauen, von älteren Menschen, von Menschen mit krankheitsbedingten Alltagseinschränkungen, von Menschen mit Migrationshintergrund und von Menschen mit geringer Bildung zu setzen.

Diese Erkenntnis ist nicht neu. Sie findet ihren Niederschlag auch bereits in zahlreichen Initiativen und Bemühungen verschiedener Akteure auf der Ebene des Bundes, der Länder, der Kommunen sowie der zivilgesellschaftlichen Organisationen. Die Ergebnisse des Freiwilligensurveys 2014 zeigen allerdings, dass die Inklusi- 
on der unterschiedlichen Bevölkerungsgruppen in das freiwillige Engagement trotz der bestehenden Initiativen bislang nicht überall gleichermaßen gut gelingt. Dies könnte daran liegen, dass es mitunter an konkreten Umsetzungsstrategien mangelt. Besonders deutlich wird dies bei der Gruppe der Menschen mit niedriger Bildung. Die Bedeutung der Bildung wird von zivilgesellschaftlichen Akteuren zwar häufig thematisiert, es werden aber nur selten systematische $\mathrm{Zu}$ gangswege für Menschen mit niedriger Bildung entwickelt. Eine Engagementpolitik, die auch darauf abzielt, die Zugangschancen gerade für bisher weniger engagierte Bevölkerungsgruppen $\mathrm{zu}$ verbessern, sollte hier ansetzen und fragen, wie bisherige Bemühungen verbessert und stärker auf die jeweiligen Zielgruppen zugeschnitten werden können.

$\mathrm{Zu}$ fragen ist aber auch, welchen Beitrag andere gesellschaftliche Akteure leisten können. Engagementförderung kann nicht nur ,von oben kommen, sie bedarf auch der Unterstützung durch die jeweils mittel- oder unmittelbar betroffenen Individuen. So scheint es beispielsweise wenig erfolgversprechend, wenn sich Organisationen zwar formal für Menschen mit Migrati- onshintergrund öffnen, diese aber von anderen Engagierten nicht in gleichem Maße akzeptiert werden wie Menschen ohne Migrationshintergrund. Freiwilliges Engagement ist darüber hinaus im Zusammenhang mit anderen Lebensbereichen zu sehen. So könnte es beispielsweise das freiwillige Engagement von Frauen deutlich befördern, wenn sich Haushalts- und Sorgetätigkeiten innerhalb der Familie in gleicher Weise auf die Schultern von Frauen und Männern verteilen würden. Auch hier kann die Politik Anreize und förderliche Rahmenbedingungen schaffen.

In der Stärkung des freiwilligen Engagements liegen nicht allein engagementpolitische Herausforderungen, sondern auch Chancen für die Gesellschaft. Die Beteiligung aller gesellschaftlichen Gruppen am freiwilligen Engagement nutzt allen Mitgliedern der Gesellschaft: Beteiligung am freiwilligen Engagement nutzt den Engagierten, denen die positiven Wirkungen einer Tätigkeit im freiwilligen Engagement zugutekommen. Freiwilliges Engagement möglichst vieler Menschen nutzt aber auch der Gesellschaft, deren Zusammenhalt vom Engagement der Freiwilligen gestärkt wird.

\section{Literatur}

Alisch, M., \& Kümpers, S. (2015). Soziale Ungleichheiten in der alternden Gesellschaft - Ein Überblick relevanter Themen und Befunde. Informationsdienst Altersfragen, 42(5), 3-11.

Bayerisches Staatsministerium für Arbeit und Soziales, Familie und Integration (2010). Grundsatzpapier des „Runden Tisches Bürgerschaftliches Engagement“. München: Bayerisches Staatsministerium für Arbeit und Soziales, Familie und Integration.

Böhnke, P. (2011). Ungleiche Verteilung politischer und zivilgesellschaftlicher Partizipation. Aus Politik und Zeitgeschichte, 61(1/2), 18-25.

Bundesministerium für Arbeit und Soziales (BMAS) (2013). Teilhabebericht der Bundesregierung über die Lebenslagen von Menschen mit Beeinträchtigungen. Teilhabe - Beeinträchtigung - Behinderung. Berlin: BMAS.

Bundesministerium für Bildung und Forschung (BMBF) (2014). Bildungsforschung 2020 - Herausforderungen und Perspektiven. Berlin: BMBF.

Bundesministerium für Familie, Senioren, Frauen und Jugend (BMFSFJ) (2005). Fünfter Bericht zur Lage der älteren Generation in der Bundesrepublik Deutschland. Potenziale des Alters in Wirtschaft und Gesellschaft - ein Beitrag älterer Menschen zum Zusammenhalt der Generationen. Berlin: BMFSFJ.

Bundesministerium für Familie, Senioren, Frauen und Jugend (BMFSFJ) (2013). Neue Wege - Gleiche Chancen. Gleichstellung von Frauen und Männern im Lebensverlauf. Erster Gleichstellungsbericht. Berlin: BMFSFJ. 
Bundesministerium für Familie, Senioren, Frauen und Jugend (BMFSFJ) (2015). Strategie "Gender Mainstreaming". Online: http://www.bmfsfj.de/BMFSFJ/Service/volltextsuche,did=192702.html (zuletzt abgerufen 5.2.2016).

Bundesministerium für Familie, Senioren, Frauen und Jugend (BMFSFJ) (2016). Engagementstrategie BMFSFJ. Strategische Ausrichtung der Engagementpolitik. Berlin: BMFSFJ

Bundesregierung (Hrsg.) (2010). Nationale Engagementstrategie der Bundesregierung. Berlin: Bundesregierung.

CDU Sachsen, \& SPD Sachsen (2014). Sachsens Zukunft gestalten. Koalitionsvertrag 2014 bis 2019. Dresden: CDU Sachsen, SPD Sachsen.

Dathe, D. (2011). Wie und wofür engagieren sich ältere Menschen? (Monitor Engagement Nr. 4). Berlin: Bundesministerium für Familie, Senioren, Frauen und Jugend.

Deutscher Bundestag (Hrsg.) (2012). Erster Engagementbericht - Für eine Kultur der Mitverantwortung. Bericht der Sachverständigenkommission und Stellungnahme der Bundesregierung (Bundestagsdrucksache 17/10580). Berlin: Deutscher Bundestag.

Deutscher Bundestag (2015). Kurzprotokoll der 9. Sitzung des Unterausschusses ,Bürgerschaftliches Engagement' vom 28. Januar 2015. Online: https://www.bundestag.de/blob/362490/bc5c196feeb486a7101d9e5ac6562aff/9_sitzung_ kurzprotokoll_oeff-data.pdf (zuletzt abgerufen 5.2.2016).

Deutscher Olympischer Sportbund (DOSB) (2015b). Integration durch Sport. Online: http://www.integration-durch-sport. de/de/integration-durch-sport/ (zuletzt abgerufen 5.2.2016).

Deutscher Olympischer Sportbund (DOSB) (2015a). 10. Frauen-Vollversammlung. Bericht der Vizepräsidentin Frauen und Gleichstellung. Online: http://www.dosb.de/fileadmin/fm-frauen-im-sport/Broschuere_DINA4_Bericht-FVV_20150818_Ansicht_02.pdf (zuletzt abgerufen 5.2.2016).

Habenicht, B. M., \& Pieper, A. (2013). Engagement von Menschen mit Behinderungen. Erfahrungen aus dem Projekt "Selbstverständlich Freiwillig". Hamburg: Diakonisches Werk.

Habermann, M., \& Stagge, M. (2014). Ältere Migranten und Migrantinnen in der kommunalen Versorgung. Eine Studie zur Entwicklung und Umsetzung niedrigschwelliger Angebote und zum kommunalen Integrationsmonitoring. Das Gesundheitswesen, 76-A66.

International Labour Organization (ILO) (2011). Manual on the Measurement of Volunteer Work. Genf: ILO.

Jakob, G. (2010). Infrastrukturen und Anlaufstellen zur Engagementförderung in den Kommunen. In: T. Olk, A. Klein \& B. Hartnuß (Hrsg.) Engagementpolitik. Die Entwicklung der Zivilgesellschaft als politische Aufgabe (S. 233-259). Wiesbaden: VS Verlag für Sozialwissenschaften.

Klatt, J., Walter, F., \& Bebnowski, D. (2011). Entbehrliche der Bürgergesellschaft? Sozial Benachteiligte und Engagement. Bielefeld: transcript.

Krimmer, H., \& Priemer, J. (2013). ZIVIZ-Survey 2012. Zivilgesellschaft verstehen. Berlin: Stifterverband für die Deutsche Wissenschaft.

Landtag Baden-Württemberg (2015). Gesetzentwurf der Landesregierung: Gesetz zur Verbesserung von Chancengerechtigkeit und Teilhabe in Baden-Württemberg (Drucksache 15/7555). Stuttgart: Landtag.

Matzat, J. (2010). Ehrenamtliches Engagement, kollektive Selbsthilfe und politische Beteiligung im Gesundheitswesen. In: T. Olk, A. Klein \& B. Hartnuß (Hrsg.) Engagementpolitik. Die Entwicklung der Zivilgesellschaft als politische Aufgabe (S. 547-570). Wiesbaden: VS Verlag für Sozialwissenschaften.

Ministerium für Arbeit und Sozialordnung, Familie, Frauen und Senioren (2014). Engagementstrategie Baden-Württemberg-Lebensräume zu „Engagement-Räumen“ entwickeln. Stuttgart: Ministerium für Arbeit und Sozialordnung, Familie, Frauen und Senioren.

Morrow-Howell, N., Hong, S.-I., \& Tang, F. (2009). Who benefits from volunteering? Gerontologist, 49, 91-102.

Olk, T., \& Hartnuß, B. (2011). Bürgerschaftliches Engagement. In: T. Olk \& B. Hartnuß (Hrsg.) Handbuch Bürgerschaftliches Engagement (S. 145-161). Weinheim: Beltz Juventa.

Rindt, S., Klein, L., \& Klein, A. (2011). Editorial. Zu viel Zivilgesellschaft? Soziale Arbeit und bürgerschaftliches Engagement. Forschungsjournal Soziale Bewegungen, 24(3), 13-18.

Schäfer, A., \& Schoen, H. (2013). Mehr Demokratie, aber nur für wenige? Der Zielkonflikt zwischen mehr Beteiligung und politischer Gleichheit. Leviathan, 41(1), 94-120. 
Schumacher, J. (2015). Kooperation von Haupt- und Ehrenamtlichen in Pflege, Sport und Kultur. Berlin: Bundesministerium für Familie, Senioren, Frauen und Jugend.

Simonson, J., Hagen, C., Vogel, C., \& Motel-Klingebiel, A. (2013). Ungleichheit sozialer Teilhabe im Alter. Zeitschrift für Gerontologie und Geriatrie, 46(5), 410-416.

Weiss, K. (2013). Migrantenorganisationen und Staat. Anerkennung, Zusammenarbeit, Förderung. In: Friedrich-EbertStiftung (Hrsg.) Migrantenorganisationen. Engagement, Transnationalität und Integration (S. 21-30). Berlin: FriedrichEbert-Stiftung.

Winker, G., \& Degele, N. (2009). Intersektionalität. Bielefeld: transcript.

Zimmer, A., \& Backhaus-Maul, H. (2012). Engagementförderung vor Ort - Was gilt es in den Blick zu nehmen? Eine Arbeitshilfe für lokale Entscheidungsträger. Münster: Westfälische Wilhelms-Universität Münster.

Zimmer, A., \& Rauschenbach, T. (2011). Bürgerschaftliches Engagement unter Druck? Einleitung. In: T. Rauschenbach \& A. Zimmer (Hrsg.) Bürgerschaftliches Engagement unter Druck? Analysen und Befunde aus den Bereichen Soziales, Sport und Kultur (S. 11-28). Opladen: Barbara Budrich.

Open Access Dieses Kapitel wird unter der Creative Commons Namensnennung 4.0 International Lizenz (http://creativecommons.org/licenses/by/4.0/deed.de) veröffentlicht, welche die Nutzung, Vervielfältigung, Bearbeitung, Verbreitung und Wiedergabe in jeglichem Medium und Format erlaubt, sofern Sie den/die ursprünglichen Autor(en) und die Quelle ordnungsgemäß nennen, einen Link zur Creative Commons Lizenz beifügen und angeben, ob Änderungen vorgenommen wurden.

Die in diesem Kapitel enthaltenen Bilder und sonstiges Drittmaterial unterliegen ebenfalls der genannten Creative Commons Lizenz, sofern sich aus der Abbildungslegende nichts anderes ergibt. Sofern das betreffende Material nicht unter der genannten Creative Commons Lizenz steht und die betreffende Handlung nicht nach gesetzlichen Vorschriften erlaubt ist, ist für die oben aufgeführten Weiterverwendungen des Materials die Einwilligung des jeweiligen Rechteinhabers einzuholen. 